\title{
HAUSDORFF DIMENSION, ANALYTIC SETS AND TRANSCENDENCE
}

\author{
G. A. EDGAR AND CHRIS MILLER
}

ABstract. Every analytic real closed proper subfield of $\mathbb{R}$ has Hausdorff dimension zero. Equivalently, every analytic set of real numbers having positive Hausdorff dimension contains a transcendence base for $\mathbb{R}$.

How large can a proper subfield of the real numbers be? Of course, before attempting to answer this question, we must make sense of what we should mean by "large" - cardinality, measure, Baire category, and so on-but there is another implicit question: What kinds of subfields of $\mathbb{R}$ should we consider? Set-theoretic independence issues quickly arise if we do not narrow the focus. We show in this note that proper subfields of $\mathbb{R}$ that are well behaved in certain algebraic and descriptive set-theoretic senses (to be made precise below) are quite small when viewed measure theoretically, although every uncountable such field has the cardinality of the continuum.

Given $E \subseteq \mathbb{R}$ and $n \in \mathbb{N}$, we write $E^{n}$ for the $n$-fold Cartesian product $E \times E \times \cdots \times E$.

A subset of $\mathbb{R}^{n}$ is analytic (also called Souslin or Suslin in the literature) if it is the continuous image of a Borel subset of $\mathbb{R}$. (There are several equivalent definitions; this one is perhaps the easiest to state.) The collection of all analytic subsets of $\mathbb{R}^{n}$ properly contains the collection of all Borel subsets of $\mathbb{R}^{n}$. Analytic sets are Lebesgue measurable and have the property of Baire. Every uncountable analytic set contains a nonempty perfect set and thus has the cardinality of the continuum. Basic information on analytic sets can be found in Cohn [2, Ch. 8] or Rogers [11]; see Kechris [8] for an extensive modern development.

Suppose that $G$ is a proper additive subgroup of $\mathbb{R}$. It follows immediately from elementary results (see e.g. Oxtoby $[10,4.8]$ ) that if $G$ is Lebesgue measurable then it has measure zero, and if $G$ has the property of Baire then it is of Baire first category. Hence, proper analytic subgroups of $(\mathbb{R},+)$ are small if we restrict our attention to Lebesgue measure and Baire category. On the other hand, any uncountable analytic subgroup of $\mathbb{R}$ has the cardinality of $\mathbb{R}$. Finer tools are needed in order to understand the situation better.

For $s \geq 0$, the Hausdorff $s$-measure of $A \subseteq \mathbb{R}^{n}$, denoted by $\mathcal{H}^{s}(A)$, is defined as follows: For $\delta>0$, let $\mathcal{H}_{\delta}^{s}(A) \in[0, \infty]$ be the infimum of all sums of the form $\sum_{j \in \mathbb{N}}\left[\operatorname{diameter}\left(A_{j}\right)\right]^{s}$ where $\left\{A_{j}\right\}_{j \in \mathbb{N}}$ is a collection of subsets of $\mathbb{R}^{n}$ with $A \subseteq \bigcup_{j \in \mathbb{N}} A_{j}$ and diameter $\left(A_{j}\right) \leq \delta$ for all $j \in \mathbb{N}$. Now put $\mathcal{H}^{s}(A)=\lim _{\delta \rightarrow 0^{+}} \mathcal{H}_{\delta}^{s}(A)$. The Hausdorff dimension of $A \subseteq \mathbb{R}^{n}$, denoted by $\operatorname{dim}_{\mathcal{H}} A$, is the infimum of all $s \geq 0$ such that $\mathcal{H}^{s}(A)=0$. (See any of Edgar [5], Falconer [7], Mattila [9] or [11] for details).

From now on, "dimension" means "Hausdorff dimension".

An ordered field $K$ is real closed if every positive element has a square root in $K$ and every one-variable odd degree polynomial function with coefficients from $K$ has a root in $K$ (equivalently, if the ring $K[X] /\left(X^{2}+1\right)$ is an algebraically closed field). Real closed

Date: June 8, 2001.

To appear in Real Analysis Exchange. 
fields play a role in the theory of ordered fields analogous to that of algebraically closed fields in the theory of fields of characteristic zero.

Here is the main result of this note:

Theorem. Every analytic real closed proper subfield of $\mathbb{R}$ has dimension 0 .

Actually, we shall prove that no analytic set $E \subseteq \mathbb{R}$ with $\operatorname{dim}_{\mathcal{H}} E>0$ is contained in any proper real closed subfield of $\mathbb{R}$. (Another way of saying this is that $E$ contains a transcendence base for $\mathbb{R}$, that is, a maximal algebraically independent subset of $\mathbb{R}$.) The converse does not hold: There are dimension 0 analytic subsets of $\mathbb{R}$ that are not contained in any proper real closed subfield of $\mathbb{R}$. Indeed, there are dimension 0 compact sets $C \subseteq \mathbb{R}$ such that the sum set $\{x+y: x, y \in C\}$ has interior, so $C$ is not even contained in any proper additive subgroup of $\mathbb{R}$. (For example, put $C=E \cup F$ where $E$ and $F$ are as in [7, Example 7.8]).

We came to the theorem while considering an open question about the possible Hausdorff dimensions of subrings of $\mathbb{R}$ that are Borel. It is well known that every proper additive subgroup of $\mathbb{R}$ is either cyclic (that is, of the form $r \mathbb{Z}$ for some $r \in \mathbb{R}$ ) or is dense and co-dense in $\mathbb{R}$. By what we noted earlier, if such a subgroup is Borel (as a subset of $\mathbb{R}$ ) then it has Lebesgue measure zero and is of Baire first category. What are the possible values for its Hausdorff dimension? It turns out that the situation is quite wild: For each $d \in[0,1]$ there is a subgroup $G$ of $(\mathbb{R},+)$ such that $G$ is Borel and $\operatorname{dim}_{H} G=d$; see Erdös and Volkmann [6] or [7, $\$ 12.4]$.

A natural variant of the question is this: What are the possible Hausdorff dimensions for subrings of $\mathbb{R}$ that are Borel? Only a partial answer is known at present: The dimension of such a ring is either equal to 1 or at most $1 / 2$. But no examples are known other than dimensions 0 and 1. A similar question for subfields of $\mathbb{R}$ that are Borel is also open. The same questions are open even when the subring or subfield is not required to be Borel but merely analytic. (See e.g. [9, pp. 166-7] for more information.)

Thus, we come to the question answered in this note: A real closed subfield of $\mathbb{R}$ that is a Borel set (or even an analytic set) has Hausdorff dimension either 0 or 1 . Moreover, dimension 1 occurs only for $\mathbb{R}$ itself.

The theorem is immediate from the following four lemmas (each of independent interest).

Lemma 1. Let $E \subseteq \mathbb{R}$ be compact, $\operatorname{dim}_{\mathcal{H}} E>0$. Then there exist $n \in \mathbb{N}$ and an $\mathbb{R}$-linear function $T: \mathbb{R}^{n} \rightarrow \mathbb{R}$ such that $T\left(E^{n}\right)$ has interior $($ in $\mathbb{R})$.

Proof. Put $\operatorname{dim}_{\mathcal{H}} E=d>0$. Choose $k \in \mathbb{N}$ such that $k d>1$; then $\operatorname{dim}_{\mathcal{H}}\left(E^{k}\right) \geq k d>1$ (see $[9,8.10])$. Hence, there is an orthogonal projection $\pi: \mathbb{R}^{k} \rightarrow \mathbb{R}$ such that the image $\pi E$ has positive Lebesgue measure [9, Ch. 9]. (In $\mathbb{R}$, Hausdorff 1-measure is the same as Lebesgue measure). Then the difference set $\{a-b: a, b \in \pi E\}$ has interior; see [10,4.8]. Put $n=2 k$ and define $T: \mathbb{R}^{n} \rightarrow \mathbb{R}$ by $T\left(x_{1}, \ldots, x_{n}\right)=\pi\left(x_{1}, \ldots, x_{k}\right)-\pi\left(x_{k+1}, \ldots, x_{n}\right)$.

Lemma 2. Let $E \subseteq \mathbb{R}$ be analytic, $\operatorname{dim}_{\mathcal{H}} E>0$. Then there exist $n \in \mathbb{N}$ and an $\mathbb{R}$-linear function $T: \mathbb{R}^{n} \rightarrow \mathbb{R}$ such that $T\left(E^{n}\right)$ has interior.

Proof. Since $E$ is analytic, it contains a compact set of positive dimension [5, (1.7.11)]. Apply the previous lemma.

Remark. Suppose, in Lemma 2, that $E$ is moreover an additive subgroup of $\mathbb{R}$. Then $T\left(E^{n}\right)$ is an additive subgroup of $\mathbb{R}$ that has interior in $\mathbb{R}$. Hence, $T\left(E^{n}\right)=\mathbb{R}$, that is, there exist 
$s_{1}, \ldots, s_{n} \in \mathbb{R}$ such that $\mathbb{R}$ is equal to the set all sums $\sum_{i=1}^{n} s_{i} e_{i}$ with $e_{1}, \ldots, e_{n} \in E$. (In particular, if $E$ is also a subring of $\mathbb{R}$, then $\mathbb{R}$ is finitely generated as an $E$-module.) This shows that proper analytic subgroups of $\mathbb{R}$ having positive dimension are, in an algebraic sense, almost all of $\mathbb{R}$.

Before proceeding further, we need some definitions and basic facts from real algebraic geometry. (See Bochnak et al $[1$, Chs. 1,2] or van den Dries [4, Chs. 2,3] for facts used below.)

A semialgebraic set $S \subseteq \mathbb{R}^{n}$ is a finite union of sets of the form

$$
\left\{x \in \mathbb{R}^{n}: p(x)=0, q_{1}(x)<0, \ldots, q_{l}(x)<0\right\}
$$

where $p, q_{1}, \ldots, q_{l}: \mathbb{R}^{n} \rightarrow \mathbb{R}$ are real polynomial functions. We say that $S$ is defined over a subfield $K \subseteq \mathbb{R}$ if every coefficient occuring in the description of $S$ belongs to $K$

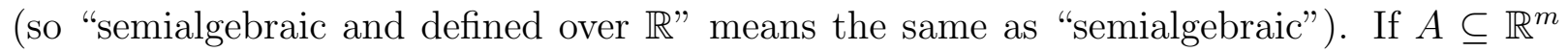
then a map $f: A \rightarrow \mathbb{R}^{n}$ is said to be semialgebraic (and defined over $K$ ) if its graph $\{(x, f(x)): x \in A\}$ is a semialgebraic (and defined over $K$ ) subset of $\mathbb{R}^{m+n}$.

Semialgebraic sets play a role in real algebraic geometry analogous to that of constructible sets in complex algebraic geometry.

Lemma 3. Let $E \subseteq \mathbb{R}$ be analytic and $K$ be the smallest real closed subfield of $\mathbb{R}$ containing $E$. Then $K$ is analytic.

Proof. The field $K$ is equal to the union of all sets of the form $f\left(E^{n}\right)$ where $n \in \mathbb{N}$ and $f: \mathbb{R}^{n} \rightarrow \mathbb{R}$ is semialgebraic and defined over $\mathbb{Q}$. (Aside: There are more explicit ways of producing $K$ from $E$ but we do not need them here.) Let $n \in \mathbb{N}$ and $f: \mathbb{R}^{n} \rightarrow \mathbb{R}$ be semialgebraic. By the cell decomposition theorem, there is a finite partition of $\mathbb{R}^{n}$ into locally closed sets $C_{1}, \ldots, C_{k}$ such that each restriction $f \mid C_{i}: C_{i} \rightarrow \mathbb{R}$ is continuous. Intersections of analytic sets are again analytic, so each $C_{i} \cap E^{n}$ is analytic. Continuous images of analytic sets are analytic, so each $f\left(C_{i} \cap E^{n}\right)$ is analytic. For each $n \in \mathbb{N}$ there are only countably many semialgebraic functions $\mathbb{R}^{n} \rightarrow \mathbb{R}$ that are defined over $\mathbb{Q}$. Countable unions of analytic sets are analytic, so $K$ is analytic.

Remark. Lemma 3 fails with "Borel" in place of "analytic" even if $E$ is also a subring of $\mathbb{R}$. However, if $E$ is a Borel subfield of $\mathbb{R}$, then $K$ is Borel. (These results were produced by R. Dougherty in personal communication with the second author.)

Remark to model theorists. An easy modification of the proof of Lemma 3 shows that if $\mathfrak{R}$ is an o-minimal expansion of the structure $(\mathbb{R},<,+, 1)$ in a countable language and $E \subseteq \mathbb{R}$ is analytic then the definable closure of $E$ - taken with respect to $\operatorname{Th}(\mathfrak{R})$ - is again analytic.

Finally, we state without proof a special case of a model-theoretic result; see [3, Lemma 4.1].

Lemma 4. Let $K$ and $L$ be real closed subfields of $\mathbb{R}$ such that $K$ is properly contained in L. Let $n \in \mathbb{N}$ and $f: \mathbb{R}^{n} \rightarrow \mathbb{R}$ be semialgebraic and defined over $L$. Then $f\left(K^{n}\right)$ has empty interior in $L$.

The point is that coefficients from $L$ are allowed in the description of $f$. The result is rather trivial if $f$ is defined over $K$, for then $f\left(K^{n}\right) \subseteq K$, and $K$ has no interior in $L$.

Proof of the Theorem. Let $E \subseteq \mathbb{R}$ be analytic with $\operatorname{dim}_{\mathcal{H}} E>0$. Let $K$ be the smallest real closed subfield of $\mathbb{R}$ containing $E$; then $K$ is analytic (by Lemma 3 ) and $\operatorname{dim}_{\mathcal{H}} K>0$. 
By Lemma 2, there exist $n \in \mathbb{N}$ and an $\mathbb{R}$-linear (hence semialgebraic) function $T: \mathbb{R}^{n} \rightarrow \mathbb{R}$ such that $T\left(K^{n}\right)$ has interior. Hence (by Lemma 4) we have $K=\mathbb{R}$.

We don't know how optimal is the theorem. Lemma 4 says that all proper real closed subfields of $\mathbb{R}$ are small in a model-theoretic sense. Lemma 3 can be regarded more generally as saying that the smallest real closed subfield of $\mathbb{R}$ containing a given $E \subseteq \mathbb{R}$ is neither much larger nor much more complicated than is $E$. But the tools needed in order to obtain Lemma 2 do not extend in general to non-analytic sets.

\section{REFERENCES}

[1] J. Bochnak, M. Coste, and M.-F. Roy, Real algebraic geometry, Ergeb. Math. Grenzgeb. (3), vol. 36, Springer, 1998, translated from the 1987 original, revised by the authors.

[2] D. Cohn, Measure theory, Birkhauser, 1980.

[3] L. van den Dries, Dense pairs of o-minimal structures, Fund. Math. 157 (1998), 61-78.

[4] _ Tame topology and o-minimal structures, London Math. Soc. Lecture Note Ser., vol. 248, Cambridge Univ. Press, Cambridge, 1998.

[5] G. Edgar, Integral, probability and fractal measure, Springer, 1998.

[6] P. Erdős and B. Volkmann, Additive Gruppen mit vorgegebener Hausdorffscher Dimension, J. Reine Angew. Math. 221 (1966), 203-208.

[7] K. Falconer, Fractal geometry: Mathematical foundations and applications, John Wiley \& Sons, 1990.

[8] A. Kechris, Classical descriptive set theory, Grad. Texts in Math., vol. 156, Springer-Verlag, 1995.

[9] P. Mattila, Geometry of sets and measures in euclidean spaces, Cambridge Stud. Adv. Math., vol. 44, Cambridge Univ. Press, 1995.

[10] J. Oxtoby, Measure and category, 2nd ed., Grad. Texts in Math., vol. 2, Springer, 1980.

[11] C. Rogers, Hausdorff measures, Cambridge Univ. Press, 1970.

Department of Mathematics, The Ohio State University, Columbus, Ohio 43210

E-mail address: edgar@math.ohio-state.edu

$U R L:$ http://www.math.ohio-state.edu/ ${ }^{\sim}$ edgar

Department of Mathematics, The Ohio State University, Columbus, Ohio 43210

E-mail address: miller@math.ohio-state.edu

URL: http://www.math.ohio-state.edu/ ${ }^{\sim m i l l e r}$ 